REVISTA ECONOMÍA

Vol. 69, N. ${ }^{\circ}$ Io9 (mayo), 99-IIo

\title{
ECONOMÍA EN LAS COMUNIDADES NOSÓTRICAS MAYA-TOJOLABALES DEL SUR-ESTE DE MÉXICO
}

\author{
VIOLETA NÚÑEZ \\ Universidad Autónoma Metropolitana, Unidad Xochimilco, México
}

Recepción manuscrito: 6 de enero de 2017

Aceptación versión final: 15 de abril de 2017

\begin{abstract}
RESUMEN Las comunidades maya-tojolabales de Chiapas en México, poseen una cosmovisión particular que nos muestran que todo en este mundo tiene vida y corazón. Es decir, conciben una sociedad repleta de sujetos — no objetos-, a partir de la cual se establece una relación intersubjetiva. Cada uno de estos sujetos humanos y no humanos —entre ellos la Madre Tierra-, forma parte de las comunidades nosotricas, en donde el fin es el bien de todos. Con esta idea edifican una economía muy particular que hoy quizá se presenta como una alternativa ante la crisis, sobre todo la alimentaria y ambiental, que enfrenta la humanidad en su conjunto.
\end{abstract}

PALABRAS CLAVE Tojolabales, cosmovisión, sujetos, nosótricas, economía.

ABSTRACT The Mayan-Tojolabal communities of Chiapas in Mexico have a particular worldview that shows us that everything in this world has life and heart. That is, they conceive a society full of subjects - and not objects-, from which an inter-subjective relationship is established. Each of these human and nonhuman subjects - among them Mother Earth-, is part of the nosotric communities, where the end is the good of all. With this idea, they build a very particular economy which today presents itself as an alternative to the crisis, especially the food and environmental crisis, which faces humanity as a whole.

KEYWORDS Tojolabales, cosmovision, subjects, nosotricas, economy.

JEL CODES Q24, Q56, R14, R30, P58.

\section{INTRODUCCIÓN}

Durante la década de los años noventa del siglo xx se vivieron diversos acontecimientos en América Latina que hicieron factible reposicionar de manera particular a los pueblos originarios. Uno de ellos, que vio nacer la década, fue la denominada «marcha indígena por el territorio y la dignidad» que partió del departamento del Beni en Bolivia, recorriendo 640 kilómetros. Otro de estos acontecimientos fue el levantamiento indígena en Ecuador, que fue nombrado como «el levantamiento de los hijos del sol», que dio paso a la «irrupción de lo indio». Junto a estos sucesos, un momento histórico fue la conmemoración de los 500 años 
del «descubrimiento de América», que aglutinó a diferentes actores de diversos países en la llamada campaña continental «50o años de resistencia indígena, negra y popular». De manera generalizada se rechazaba el «descubrimiento», argumentándose que desde la llegada de los europeos, cinco siglos atrás, se habían negado las culturas de los pueblos que ya habitaban el continente, el llamado Abya Yala.

La negación del otro, de la otredad, daba paso a hablar de «en-cubrimiento» (Dussel, 1992) y no de «descubrimiento». Junto a estos procesos sociales, en México se vivió un hecho histórico que daría paso a la gestación de un proceso que no ha terminado. Este acontecimiento fue la rebelión de indígenas y campesinos del sur-este mexicano, quienes, levantados en armas, declararon la guerra al gobierno mexicano y dijeron ¡ya basta! de la pobreza y marginación de la que habían sido objeto durante 500 años, ante lo cual demandaban, entre otras cosas, libertad y democracia. Entre estos indígenas, que conformaban el Ejército Zapatista de Liberación Nacional, se encontraban junto con los pueblos mayas tseltal, tsotsil, cho'l, los tojolabales, uno de los más de 60 pueblos indígenas que habitan en la república mexicana.

Desde la cosmovisión de este pueblo maya, todo tiene vida y corazón, es decir, el mundo está repleto de sujetos, lo cual permite relaciones intersubjetivas. Pero además, estos sujetos forman parte de la comunidad, y de lo que ellos nombran el «nosotros», las comunidades nosótricas. Esto los lleva a prácticas de vida específicas, cosmovivencias, entre ellas la forma de ejercer y practicar su economía, que puede llevar a hablar de una economía nosótrica, la cual abordaremos en las siguientes líneas y sobre la cual me interesa preguntarme si ¿la economía nosótrica de los maya-tojolabales constituye una alternativa frente a la economía dominante - basada en la extracción de recursos y quema de restos fósiles- y ante la crisis civilizatoria que estamos viviendo, crisis que pone en peligro al planeta en su conjunto?

\section{LOS MAYA-TOJOLABALES}

Los maya-tojolabales habitan principalmente en el estado de Chiapas en México, un estado que se encuentra en el sur-este de la nación y que albergó desde antes del proceso de conquista y colonización española a la cultura maya. Como parte de la historia de los tojolabales, resalta el hecho de que vivieron como peones acasillados en las fincas del sur-este mexicano, en donde a cambio de un pedazo de tierra o habitación, los peones pagaban en especie o con un servicio. ${ }^{1}$ El trabajo que allí realizaron fue un trabajo que los marcó como pueblo en su conjunto. Primero, porque los golpes que recibieron durante el acasillamiento dejaron marcas físicas en sus cuerpos por los golpes y castigos que recibieron en las fincas. Pero también el intenso y cruel trabajo, tanto de hombres como mujeres, y en medio de ello tener que cargar a los patrones y a sus familias en la espalda - amarrándose una silla, literalmente como si fueran «mulas de carga»- por largas distancias y por caminos inhóspitos.

A esto se suma la no remuneración del trabajo en la finca, que los llevó a nombrar a esta etapa como la época del baldío, porque el trabajo se hacía en balde, para el patrón, sin recibir retribución alguna. Este hecho también los dejó tatuados. No hay tojolabal alguno, hasta hoy en día, que no hable de esta fase de su historia. Una historia muy reciente porque algunos de ellos pudieron salir con dificultades de la finca y del acasillamiento hasta el año de 1994, 
cuando se levantó en armas el EZLN y lograron tomar y recuperar tierras para lograr liberarse de la finca - de donde era muy difícil salir por deudas o por represión-. Al respecto, durante los primeros meses del levantamiento se recuperaron más de 100.000 hectáreas (Gobierno del Estado de Chiapas, 1997), hecho que les permitió establecerse como poseedores de la tierra algunos fueron reconocidos como ejidos-.

Aunado a su particular historia, este pueblo indígena posee una cosmovisión muy peculiar que es parte de la herencia de la visión del mundo de los pueblos mayenses. Para los tojolabales, todo lo que habita en este mundo es un sujeto con vida y corazón. Entre ellos los que habitan el mundo terrestre; los que habitan el mundo celeste y los que se encuentran en el inframundo, los «muertos», que, desde su visión del mundo, siguen siendo sujetos importantes en la comunidad, incluso hay que indicar que la palabra corazón en tojolabal se dice altsil, y los «muertos» son nombrados como altsilal.

\section{COMUNIDADES NOSÓTRICAS}

Para los tojolabales, todo lo que está en el mundo o en los mundos - terrestre, celeste e inframundo- como ellos lo dividen, además de ser un sujeto, forma parte fundamental de las comunidades. A partir de esta idea, las relaciones que se establecen con «todo» lo que existe en los mundos, son relaciones entre sujetos, intersubjetivas, y no entre objetos (Lenkersdorf, 2002). Pero además, los diferentes sujetos constituyen el «nosotros». Y en este nosotros, dicen los tojolabales, hay una visión de totalidad. Primero, porque contempla a los ancianos, ancianas, hombres, mujeres, niños y niñas. Segundo, porque también incluye a todos los otros sujetos no humanos. Al respecto, los tojolabales conciben al territorio como un territorio que abarca tres mundos: el mundo terrestre -lu'um k'inal_-, el mundo celeste - sat k'inal- y el inframundo $-k^{\prime} i k$ kinal- En cada uno de estos mundos, habitan los sujetos que forman parte del nosotros.

En el primero de los mundos, en el terrestre, se encuentra uno de los principales sujetos, y que es fundamental en la economía de los tojolabales, la tierra, la Madre Tierra, nombrada por los tojolabales como la jnantik lu’um - nuestra Madre Tierra-. Pero también en este mundo habitan los árboles y bosques — considerados como guardianes de las comunidades-, plantas, flores, animales, manantiales, ríos, mares, lagunas, piedras, entre otros. En el segundo de los mundos, el celeste, está habitado por otros de los sujetos de suma importancia, el Sol, considerado como el Padre Sol, y la Luna, también de gran relevancia, al ser nombrada como la Madre Luna - nan ixaw-.

Asimismo, en este mundo habitan las estrellas, las nubes y los rayos. Por su parte, en el mundo de «abajo», habitan los «muertos», a quienes se les consulta sobre los temas relevantes de la comunidad. Los sujetos que viven en el inframundo, los «muertos», son llamados como altsilales, siendo que altsil en lengua tojolabal significa corazón, lo cual evidencia la visión que tienen de los que pasan a habitar este otro mundo, «muertos con corazón». Así, todos los que viven en los diferentes mundos, desde la perspectiva tojolabal, tiene vida y corazón, y son sujetos elementales en las comunidades nosótricas. 


\section{ECONOMÍA DEL NOSOTROS}

La palabra economía tiene como origen la palabra griega, oikonomía. Oikos significa casa, morada o ámbito vital. Por su parte, nomía, del griego nómos, significa ley, norma. Así, la economía tendría como significado «las leyes o normas de la casa o del lugar donde se habita». Pero también oikonomía, es traducido como oîkos — casa- y némein — distribuir, administrar-, lo que implicaría que la economía también se refiere a la «distribución o administración de la casa». Esta concepción se acercaría más al concepto de economía política, que estudia y analiza el modo de producción, distribución y consumo en una sociedad, y sus relaciones sociales que se establecen. En este sentido, analizaremos cómo son las «normas de la casa» para producir, distribuir y consumir en esta sociedad que concibe a todo lo que está a su alrededor como un sujeto, y como parte del nosotros, lo que constituye las sociedades nosótricas. Pero también veremos a grandes rasgos qué y cómo producen y consumen.

\section{PRODUCCIÓN: MADRE TIERRA}

La tierra es un sujeto fundamental para los tojolabales. Para ellos, al igual que para otros pueblos indígenas, representa la madre. Es el sujeto que les da de comer, los alimenta, los nutre, los protege. En síntesis, por ella, la Madre Tierra, es posible vivir y su estancia en el mundo. Esto se dice y se cuenta de manera cotidiana en las comunidades, y ha quedado plasmado en fragmentos de poemas tojolabales (Lenkersdorf, 1998, p. 92). De la tierra, este pueblo maya obtiene su alimento principal, el maíz. De esta gramínea, hasta hoy se piensa que están formados los cuerpos de los hombres y mujeres mayas. Por eso se nombran «los hombres y mujeres de maíz», como ya se establecía desde el texto maya antiguo Popol Vuh, cuando se cuenta la historia de cómo se formó el cuerpo de los hombres en la Tierra (Raynaud, González de Mendoza y Asturias, 2000, pp. 101-102).

A la tierra, mejor dicho, a la milpa - herencia mesoamericana- acuden a sembrar además de maíz, frijol, tomate, calabaza, chile, entre otros. Con ella se establece una relación particular, porque hay que cuidarla y protegerla todos los días. Incluso hay quienes indican que se establece una relación de enamoramiento con la tierra, como con una «novia» a quien se acude a su encuentro día con día.

Es por esto que al sembrar para producir los alimentos, lo hacen respetando lo más posible a este sujeto de suma importancia. Con esta idea, indican los tojolabales, aunque en esta época la tecnología ha facilitado los trabajos de campo, por lo general las comunidades indígenas no han incorporado los avances tecnológicos, «para las tojolabales son mejores las costumbres que han dejado nuestros ancestros con el preparar las yuntas, ${ }^{2}$ los barzones ${ }^{3}$ y nuestro arado, luego viene la siembra» (Vázquez Méndez, 1998, p. 263). Es decir, se mantiene una tecnología rudimentaria, que ha permitido conservar y proteger la tierra. Así se establece una relación de mayor armonía con la naturaleza, a diferencia de la relación que se lleva a cabo con una racionalidad únicamente de ganancia, la cual impera en el régimen de acumulación capitalista. Con esta visión del mundo, con cada ciclo de siembra se lleva a cabo una fiesta a la tierra, a la Madre Tierra; en esta celebración, toda la comunidad se reúne para agradecerle los frutos de su vientre, que han hecho posible su alimentación, y para pedirle que los siga alimentando. 
Junto a esto, en la producción con la tierra emerge otro de los elementos fundamentales del pueblo tojolabal, el kisa, que traduciéndolo significa respeto, hacerle caso a alguien, reconocer, aceptar, atender, tratar bien, tomar en consideración (Lenkersdorf, 2004, p. 182); un respeto que se establece con los diferentes sujetos que conforman el nosotros, pero en particular con su Madre Tierra. Dicho en términos incorrectos, estos mayenses cuidan su «medio de producción» - por supuesto que no lo es, pero si así la concibiéramos-y piensan en el futuro, lo cual hace posible de hablar de una sociedad sustentable. Es por esto que procuran no destruir el «medio» que les posibilita producir su alimento, por el contrario, lo cuidan al extremo.

Así, el cuidado de la tierra se sustenta no solo en la visión del mundo o en el discurso, sino en las prácticas agroecológicas que mantienen al producir los alimentos de la tierra, intentando no introducir agroquímicos que puedan tener un impacto presente y futuro en este ser y en el resto de los seres que dependen de ella. En este sentido, su siembra se realiza con abonos orgánicos, sin la utilización de insecticidas y abonos químicos, y se ha ido introduciendo la práctica de roza, tumba y pica, que sustituye la práctica de la quema —última fase del proceso antes de la siembra-. Con esto, el dejar descansar a la tierra, mediante los acahuales es otra puesta en marcha, lo cual también contribuye a la búsqueda de cierto equilibrio con la naturaleza.

\section{PRODUCCIÓN: RACIONALIDAD NO CAPITALISTA}

En las comunidades maya-tojolabales, como en otras comunidades indígenas campesinas, no impera una racionalidad capitalista. Su lógica no es la acumulación de capital. Es decir, el fin de este pueblo, no es la ganancia, sino la reproducción de la vida (Shanin, 1979). Una vida no sustentada en la generación de las mercancías, producidas bajo un contexto de explotación del Hombre - producción de un plusvalor no retribuido-, cuyo fin no es el valor de uso, sino el valor de cambio y la valorización del valor. Pero además, la visión del mundo de los tojolabales hace que no exploten los recursos naturales de una manera irracional como lo hace el capital.

Es por esto que en México - un país megadiverso- la mitad de las selvas y una tercera parte de los boques están en manos de los pueblos indígenas (Robles y Concheiro, 2004). Bajo esta racionalidad, la extracción que realizan a la naturaleza se fundamenta en la reproducción de la vida, de su ser indígena y campesino y no en la reproducción del capital. Así, en cada ciclo de producción de esta sociedad nosótrica se busca recrear su modo de vida campesindio (Bartra, 2010).

\section{PRODUCCIÓN: NECESIDAD COMUNITARIA}

Con esta otra racionalidad no capitalista se siembra principalmente para el autoconsumo, lo que significa que se produce lo que se requiere para cubrir las necesidades de las familias de las comunidades y no con fines de ganar con lo producido. Es decir, no se produce para acumular. En la siembra se involucra toda la familia de diferentes maneras. Durante la siembra y en el cuidado del crecimiento del maíz, es principalmente el hombre quien se involucra, pero después, en la cosecha, participa el resto de la familia. Sin recibir pago monetario alguno, ellos saben que la tierra los alimentará y que de la milpa y los solares, de su gran diversidad de especies comestibles que en ella crecen, podrán vivir. 
En este sentido, este proceso de producción está al margen de la lógica de acumulación capitalista. Si bien es cierto que se desarrolla en un país y en continente donde el capital es la relación social que domina, los tojolabales producen con otra lógica y racionalidad. Pero además, considerando que se produce para satisfacer las necesidades de la comunidad. En este modo de producir, indicaba, no existe la explotación, pero además, no hay un salario que cubra el precio de la fuerza de trabajo y un espacio de realización de la plusvalía para convertirse en ganancia. Este trabajo es nombrado como 'a'tel y como komon 'a'tel, que hacen referencia al trabajo en común, al trabajo colectivo, pensados y elaborados para el bien común de la comunidad.

No obstante, esto no implica que estén totalmente al margen del sistema capitalista. A éste se vinculan mediante el mercado de productos y al mercado de trabajo. Del producto sembrado, venden una proporción muy pequeña a fin de comprar productos que no pueden producir al interior de sus comunidades; pero también la situación económica los ha llevado a que en ocasiones tengan que vender su fuerza de trabajo; sin embargo, no es lo común porque saben perfectamente de la discriminación, dureza y racismo a la que se tendrán que enfrentar en cualquiera de los mercados. No obstante, al acudir a estos mercados es posible cubrir necesidades no satisfechas a partir de lo que se produce en las comunidades, entre ellas, algunos productos industrializados; pero antes de acudir a éste, realizan una práctica que es nombrada como bólmal, que implica el intercambio de productos sin la mediación de dinero.

\section{DISTRIBUCIÓN: PLANEACIÓN}

Lo que se produce, en términos de cantidad, también escapa a la lógica capitalista, debido a que se siembra con cierta planeación, pensando en cubrir las necesidades de la comunidad y dejar un pequeño remanente que pueda ser intercambiado o vendido. Así, a diferencia de la producción capitalista que se realiza de manera caótica, sin planeación, y sin saber si lo que se produce se consumirá y si cubrirá las necesidades de la población en su conjunto, desde la lógica tojolabal se produce primero para satisfacer el alimento que requiere la población.

De sobra está decir que las mil millones de personas con hambre en el mundo (FAO, 2013), y las miles de toneladas de alimento que se tiran en el planeta, verifican la producción caótica y sin planeación en el régimen capitalista a la cual me refiero. Al respecto se indica que «solo las 40 millones de toneladas de alimentos despilfarrados en los EE. UU. cada año podrían alimentar a los 1000 millones de personas que se van a la cama con hambre cada día» (Stuart, Tristram, 2011). Así, además de que los datos ponen en duda que la producción en el capital traiga beneficios y desarrollo para la humanidad en su conjunto, evidencian la no planeación y el desinterés porque el común de los seres humanos vivan sin hambre, ya no hablemos de bienestar en su conjunto.

Regresando a los tojolabales, como punto de partida se piensa que no haya hambre. Es por esto que en momentos de dificultad de algunas de las familias que integran las comunidades nosótricas, éstas son apoyadas por el conjunto de los integrantes, ya sea compartiéndoles alimento o apoyando en la siembra de sus parcelas, ya que se busca el «bienestar del nosotros» en el que la comida, el maíz, es parte de su fundamento. Con esta visión, este pueblo mayense tiene una idea de pobreza asociada no a los bienes materiales, sino al no tener comida. Desde 
su pensamiento, pobre lo nombran como mey swa’el, cuya traducción podría ser «no tiene comida». Así, la pobreza no está relacionada con tener cosas materiales, sino con lo que conforma y edifica su cuerpo.

Con esta creencia, la producción del alimento debe de alcanzar al conjunto de la comunidad, porque de lo contrario, no tendrán un lekilaltik, un «bien de nosotros», un bien común, que en fechas recientes ha sido traducido como el «buen vivir» tojolabal.

\section{DISTRIBUCIÓN: TRUEQUE (B'OLMAL)}

Desde la época prehispánica, el pueblo tojolabal lleva a cabo intercambios de productos, de valores de uso entre las diferentes comunidades sin mediar ningún tipo de dinero. Esta práctica es nombrada como bolmal, lo que podría ser traducido como trueque. Mediante el bolmal se establecen relaciones de cierta solidaridad entre las comunidades debido a que este acto se realiza a partir de la necesidad de las comunidades, quienes se complementan entre ellas. Es decir, existe un acto de complementariedad y de lo que se nombra como "compartencia» ${ }^{4}$ en lugar de la «competencia». Para realizarlo, las comunidades siembran pensando - o planeandoque acudirán a las otras comunidades a fin de cubrir la mayor parte de sus necesidades, pero también en solidarizarse ante la carencia de los otros. El bolmal, que decía era una práctica desde antes de la llega de los españoles a este continente, no desapareció con el arribo del dinero como mercancía dominante del capital. Cabe indicar que el concepto «dinero» no existe en tojolabal, cuando se hace la traducción, se le nombra como takin, cuyo significado es metal.

Si bien ahora ya está presente la mercancía dinero, ésta no es utilizada de forma preponderante en las comunidades, ya que el principal trabajo, nombrado como 'a'tel, se realiza sin pago, ya que se concibe y se realiza como un servicio a la comunidad. Sobre el 'a’tel, Lenkersdorf indica que éste «se refiere a los trabajos en la milpa y otros trabajo en y a favor de las comunidades [...]. Todos estos tipos de trabajo se hacen para el bien común de la comunidad, es decir, que todos y cada uno tenga vida [...]. Por el 'a'tel [...] no se recibe salario alguno, sino que se hace por y para la vida comunal» (Lenkersdorf, 2004, pág 203).

Así, el trueque que implica el intercambio de productos sin la mediación del dinero, es parte de las comunidades en el siglo xxi, lo cual muestra una lógica muy particular, dirían los zapatistas, una lógica «muy otra». En este intercambio, emergen los valores de uso necesarios para las comunidades; pero además, emerge una idea de un trato de mayor horizontalidad que no ocurre en el mercado donde media el dinero. Al respecto, Casimiro un joven tojolabal indica que:

[E]s muy diferente vender en un mercado en la ciudad que cambiar en las comunidades. En la ciudad, los kaxlan(es) [los no indígenas] siempre quieren comprar más barato nuestros productos. Y como no hay acuerdo entre las comunidades, terminamos vendiendo barato nuestro producto. Pero es muy diferente cuando cambiamos nuestros productos entre comunidades [...] El que llega a hacer trueque intercambia una medida de su producto y recibe una misma medida, pero de otro producto. Pero el que llega a cambiar me da mi regalo (el pilón que nosotros decimos), entonces el que recibe también da su regalo, pero da más del que recibió, dicho de otro modo, da más pilón... para ponernos 'parejos', porque el que viene de otra comunidad a hacer trueque no lo tiene y lo necesita. (Casimiro, citado en Ayala, 2015, pp. 158-159) 
Una vez que no pueden ser cubiertas las necesidades, a partir de lo que producen entre ellas, es cuando se recurre a los mercados de productos y de trabajo, pero como he señalado, sabiendo que vivirán la ley de san garabato al revés: "comprar caro y vender barato», y, como dice Armando Bartra, entrarán a ser parte del proceso de explotación del trabajo campesino por el capital, mediante la incorporación a estos mercados, en donde son subsumidos bajo la lógica de acumulación de capital (Bartra, 1982).

CONSUMO

El consumo refleja parte del equilibrio que hay en las comunidades, sintetizado en lo que los tojolabales llaman lajan lajan aytik, que se podría traducir como estar iguales o parejos, mismo que forma parte del lekilaltik, el bien de todos, de la comunidad nosótrica. Se intenta que todos coman, por lo cual se procura que el alimento no falte. Es en este sentido que se han edificado milpas colectivas de maíz y frijol.

El consumo que realizan las comunidades tojolabales está basado principalmente en los alimentos que se cosechan de la tierra. Su consumo fundamental es el maíz y el frijol, y los productos que constituyen los traspatios o solares — café, mango, plátano, naranja, caña de azúcar, aguacate, lima, entre otros-y algunas regiones silvestres - miel-. En escasas ocasiones, en momentos o fechas particulares - fiestas o celebraciones de la comunidad- se consumen pollos o carnes rojas. A éstos se agregan los valores de uso que se adquieren en el bolmal, los cuales también emergen sustancialmente de la tierra de las comunidades. Sin embargo, en años recientes se han ido incorporando cada vez más productos industrializados, algunos muy necesarios, como artículos de aseo e higiene - champús, jabones, peines-o de primeros auxilios, telas, entre otros. Pero otros de estos productos, no de primera necesidad, han sido introducidos a las comunidades, entre ellos, frituras, refrescos, sopas de pasta, productos con edulcorantes y con harinas refinadas.

Por supuesto que la introducción de este tipo de mercancías ha traído modificaciones en los territorios. Por una parte el cuerpo de los hombres y mujeres se modifica, lo cual incluso podría traer cambios en la salud y, por la otra, al llegar los productos industrializados se observan los desechos, lo cual contribuye a contaminar y a transformar los territorios. No obstante, sigue predominando el consumo de los alimentos que se generan a partir del trabajo de la tierra, del 'a'tel. Es decir, el maíz y el frijol continúan siendo los fundamentos de este pueblo maya, lo cual también evidencia la lógica particular, mencionada en líneas anteriores.

\section{ECONOMÍA NOSÓTRICA TOJOLABAL COMO PARTE DEL PROYECTO NEOZAPATISTA}

La economía tojolabal a la que he hecho referencia, que considera a una totalidad en la que se contempla una complejidad de sujetos, forma parte del proyecto de autonomía que se constituyó en el estado de Chiapas, en particular en el territorio que habitan las comunidades y pueblos que conforman el Ejército Zapatista de Liberación Nacional. El proyecto de autonomía zapatista tomó un rumbo particular en el año 2001 cuando el gobierno federal, después de un amplio movimiento social que involucró a millones, aprobó una reforma indígena que no 
reconocía a los pueblos indígenas como sujetos de derecho y limitaba de manera importante el ejercicio pleno de la libre determinación y de su autonomía. Al respecto, los propios zapatistas lo calificaron como «una grave ofensa a los pueblos indios, a la sociedad civil nacional e internacional, y a la opinión pública, pues desprecia la movilización y el consenso sin precedente que la lucha indígena alcanzó en estos tiempos» (Subcomandante Marcos, 2001). Este hecho forzó a los zapatistas a «romper» relaciones con el gobierno federal, declararse en rebeldía - no aceptando ningún dinero de programas gubernamentales- y continuar de facto con su proceso de construcción de su autonomía.

Como parte de este proceso autonómico, las comunidades indígenas y campesinas, siguiendo la cosmovisión maya y los principios del EZLN, han optado por el trabajo colectivo. Sobre este último punto, la Primera Declaración de la Selva Lacandona de 1994, donde se declara la guerra al gobierno mexicano, se presentaron las Leyes Revolucionarias del EZLN. Algunas de estas leyes como la Ley Agraria Revolucionaria, estableció como prioridad el trabajo colectivo y cooperativo. En varios artículos de la ley se enfatizó sobre esta forma de trabajo, cuyo fin se plasmó en el artículo décimo que decía:

El objetivo de la producción en colectivo es satisfacer primeramente las necesidades del pueblo, formar en los beneficiados la conciencia colectiva de trabajo y beneficio y crear unidades de producción, defensa y ayuda mutua en el campo mexicano. Cuando en una región no se produzca algún bien se intercambiará con otra región donde sí se produzca en condiciones de justicia e igualdad. Los excedentes de producción podrán ser exportados a otros países si es que no hay demanda nacional para el producto. (EZLN, 1993)

Siguiendo este principio, y como parte del proceso de rebeldía y autonomía, en diferentes comunidades se han conformado trabajos colectivos, edificándose cooperativas, en donde se involucran tanto hombres y como mujeres. Entre ellos se han establecido milpas de maíz y frijol colectivas a fin de garantizar uno de los elementos fundamentales de la vida, la alimentación. En este sentido, pese a que a nivel nacional México ha incrementado su importación de maíz, lo cual ha provocado la pérdida de soberanía y seguridad alimentaria, el territorio zapatista ha apostado por garantizar que su población se alimente. Así, este hecho, que es uno de los cambios del proceso autonómico, muestra que la reproducción del sujeto es lo fundamental en el proyecto zapatista.

A más de esto, las comunidades tienen colectivos de otros productos que completan la dieta familiar, entre ellos los colectivos de ganado, pollos, café y miel. Pero también han edificado cooperativas agroecológicas, ganaderas, tiendas comunitarias, panaderías, artesanías, productos medicinales, herbolarios. Cabe indicar que algunos de estos colectivos están conformados únicamente por mujeres, lo cual ha contribuido a tener otro rol y responsabilidad de las tojolabales en las comunidades. Este es otro de los grandes cambios, ya que la mujer a partir de esto, puede desempeñar otro rol social. La unión y producción en colectivo, ha hecho factible cubrir las necesidades básicas de alimento, lo cual garantiza la seguridad alimentaria, y ha posibilitado que los zapatistas estén exportando algunos productos, entre ellos, café y miel a otros lugares del país e incluso a otros países como Italia, Alemania, Francia y Grecia. Al respecto, se apunta: 
Se han ido buscando pasos de cómo comercializar nuestra producción por medios justos para que sea directa nuestra comercialización, buscar acuerdos de cómo vender nuestra producción, quizás formando cooperativas, buscando redes con la gente solidaria, se ha podido hacer poco por lo difícil que es ir en contra del capitalismo pero se están haciendo todos los esfuerzos para poder vender [a un precio] justo nuestros productos. (Junta de Buen Gobierno-La Garrucha, 2006)

Como parte de su proyecto autónomo, los zapatistas también han creado el Banco Popular Autónomo Zapatista y el Banco Autónomo de Mujeres Zapatistas, cuya prioridad es prestar para atender casos de salud y para los proyectos colectivos y cooperativos. Este banco del pueblo y para el pueblo tiene una tasa de interés del dos por ciento; en fechas recientes han realizado un proceso de consulta entre sus comunidades a fin de consensuar si debe deben o no cobrar a los hombres y mujeres que solicitan un préstamo y se encuentran enfermos. Sobre este hecho un zapatista tojolabal comenta:

Algunos dicen que está mal cobrar interés para los casos en que se necesita dinero por alguna enfermedad [...] Yo creo que vamos hacer un acuerdo de nuevo de que los enfermos no pueden dar interés, pues dónde va encontrar dinero, si de por sí está muriendo. Ahorita se están consultando a los pueblos: si se cobra o no interés. (Moisés, citado en Ayala, 2015, pp. 161-162).

Así, el proyecto tojolabal que se enmarca en el proceso autonómico zapatista se circunscribe en la idea de una sociedad solidaria, que considera que las «normas de la casa», su oikos nomos - economía-, deben regirse pensando en el bien común la pluralidad de sujetos que habitan las comunidades nosótricas.

\section{A MANERA DE CONCLUSIÓN}

Ante la crisis que atraviesa la humanidad, entre ellas la económica, la migratoria, la alimentaria y la ambiental, las prácticas de las comunidades, pueblos y naciones originarias, podrían constituir una alternativa civilizatoria. No representan una utopía, estas comunidades son una realidad.

En particular, al hablar de economía, los pueblos maya-tojolabales del sur-este mexicano tienen mucho que aportar y enseñarnos. Su enseñanza se desprende de su cosmovisión que considera que todo en este mundo tiene una razón de ser. Pero además, de concebir a todo lo que está en el mundo como sujetos, lo cual nos habla de una comunidad repleta de sujetos -y no de objetos-. Con esta idea se desprende una relación intersubjetiva, es decir, todos se posicionan frente a los otros, como sus iguales. Junto a esto, los tojolabales tienen la concepción de que cada sujeto - todos los sujetos- forma parte de la complejidad del nosotros, del tik tojolabal, que constituye lo que ellos nombran como comunidades nosótricas.

Con esta cosmovivencia, que significa la puesta en práctica de su visión del mundo, llevan a cabo una economía en donde se respeta - kisa - al principal ser que los alimenta mal dicho, su «medio de producción»-y los edifica como hombres y mujeres, a la madre tierra - jnantik lu'um-; de ella se obtiene el sustento principal de este pueblo maya, el maíz. Es por esto que la cuidan, porque saben perfectamente que de ella depende su futuro y su bien común -lekilaltik-. 
Por esto, además de utilizar prácticas agroecológicas, producen lo que necesitan para alimentarse, con la intención de consumir lo que producen, sin la idea de acumular. Junto a esto, llevan a cabo prácticas como el bolmal que significa el intercambio de productos sin la intermediación del dinero que además de cubrir las necesidades insatisfechas, busca la compartencia en lugar de la competencia, lo cual muestra su idea de complementariedad.

Esto se lleva a cabo con la idea de que las comunidades, todas, puedan vivir un lajan lajan aytik, que significa estar parejos o iguales, es decir, en este caso, que todos tengan que comer y no vivir lo que ellos nombrarían como pobreza, el mey swa’el — no tiene comida-. En este sentido, han puesto gran importancia al trabajo colectivo, komon 'a'tel, que no se hace para recibir un salario, sino pensando en que las comunidades no pasen hambre.

En contraste, a esta última idea, los mil millones de hombres y mujeres que se van con hambre a la cama todos los días, me hace recordar lo que decía Lenkersdorf: ¿Qué sociedad es aquella que crea mucha riqueza, pero tiene sin comer a una buena parte de su población? Desafortunadamente, esa sociedad es la nuestra — no la que queremos_-, la capitalista, que produce millones de hambrientos en el mundo, situación que nos duele y todo los días nos hace afirmar que definitivamente el camino de la acumulación de capital, no es el camino. De allí que tenemos mucho que aprender de las economías y sociedades nosótricas.

\section{NOTAS}

1 «Este tipo de relación laboral de corte servil o señorial [...] fue común en toda Hispanoamérica donde el peonaje se generalizó en el período colonial [...]. Esta forma de servidumbre atada a la tierra en el sistema de haciendas, se conoce con diferentes nombres según los países: arrendatarios y más tarde inquilinos en Chile, terrazgueros y luego peones acasillados en México, vanaconas en la sierra del Perú y Bolivia, huasipungueros en el Ecuador y otros» (Cabrera, 2007, p. 285).

2 Par de animales - bueyes o mulas- para la siembra.

3 Instrumento que une a los bueyes o mula en la yunta.

4 Me refiero a la práctica del compartir entre los pueblos que edifican lo que en México se nombra como comunalidad.

\section{REFERENCIAS}

Ayala, C. (2015). La economía política del escuchar. En M. Millán y D. Inclán (Coords.), Lengua y cosmovisión, intersubjetividad. Acercamientos a la obra de Carlos Lenkersdorf. México DF, México: UnAM.

Cabrera, R. (2007). Tierra y ganadería en Guanacaste. San José, Costa Rica: Editorial Tecnológica de Costa Rica.

Bartra Verges, A. (1982). La explotación del trabajo campesino por el capital. México DF, México: Macehual.

Bartra Verges, A. (2010). Campesindios. Aproximaciones a los campesinos de un continente colonizado. La Paz, Bolivia: Instituto para el Desarrollo Rural de Sudamérica-Oxfam.

Von Houwald, G. F. (1979). Nicolás de Valenzuela: Conquista del Lacandón y Conquista del Chol. Relación sobre la expedición de 1695 contra los Lacandones e Itzá según el «Manuscrito de Berlín» (Biblioteca Ibero-Americana). Berlín, Alemania: Colloquium Verlag.

Dussel, E. (1992). 1492. El encubrimiento del otro. Hacia el origen del mito de la modernidad. Madrid, España: Nueva Utopía. 
Ejército Zapatista de Liberación Nacional (1993). Ley Agraria Revolucionaria. Palabras. Recuperado de http://palabra.ezln.org.mx/comunicados/1994/1993_12_f.htm

Gobierno del Estado de Chiapas (1997). Diagnóstico de la situación agraria de Chiapas. Chiapas, México. Secretaría de Desarrollo Agrario.

González Casanova, P. (1998). Cuentos y relatos indígenas. México DF, México: UNAM.

Jiménez, R. (2001). La niña y la sagrada tierra. En V. Gutiérrez (Dir.), Ja bajlami wax yala. Selección de cuentos tojolabales. Chiapas, México: Tuxtla Gutiérrez.

Junta de Buen Gobierno-La Garrucha (2006). Informe sobre «Lo que se ha hecho en proyectos de comunidades zapatistas». En Baronnet, Mora y Stahler-Sholk, Luchas "muy otras". Zapatismo y autonomía en las comunidades indígenas de Chiapas. México DF, México: Universidad Autónoma Metropolitana.

Lenkersdorf, C. (1998). Tojol 'ixuk winikotik. 'oj tz’eb' anukotik. México DF, México: Centro de Reflexión Teológica.

Lenkersdorf, C. (2002). Filosofar en clave tojolabal. México DF, México: Miguel Ángel Porrúa.

Lenkersdorf, C. (2004). Conceptos tojolabales de filosofía y del altermundo. México DF, México: Plaza y Valdés.

Lenkersdorf, G. (1986). Contribuciones a la historia colonial de los tojolabales. En M. H. Ruz, Los legítimos hombres. Aproximaciones antropológicas al grupo tojolabal. México DF, México: UnAM.

Muñoz, G. (2013, agosto 10). La autonomía zapatista en perspectiva social. Regeneración radio. Recuperado de http://www.regeneracionradio.org/ index.php/autonomia/autonomia/ item/3984-la-autonom\%C3\%ADa-en-perspectiva-social

Organización de las Naciones Unidas para la Alimentación y para la Agricultura (fAO) (2012). Pérdidas $y$ desperdicio de alimentos en el mundo. Alcance, causas y prevención. Roma, Italia: FAO.

Organización de las Naciones Unidas para la Alimentación y para la Agricultura (FAO) (2013). La FAO acoge el debate «Mil millones de hambrientos: ¿Podemos alimentar al mundo?». FAO. Recuperado de http://www.fao.org/news/story/es/item/170818/icode/

Raynaud, G., González de Mendoza J. M. y Asturias, M. A. (Traductores) (2000). Popol Vuh. El libro del consejo. México DF, México: unam.

Robles, H. y Concheiro, L. (2004). Entre las fábulas y la realidad, los ejidos y las comunidades con población indígena. México DF, México: UnAM.

Shanin, T. (1976). Naturaleza y lógica de la economía campesina. Barcelona, España: Anagrama.

Stuart, T (2011). Despilfarro. El escándalo global de la comida. oxfam Intermón. Recuperado de http:// www.oxfamintermon.org/es/editorial/entrevista-se-desperdicia-mas-comida-en-mundo-deque-podrian-consumir-todas-personas-hambr

Subcomandante Marcos (2001). Comunicado del Comité Clandestino Revolucionario IndígenaComandancia General del Ejército Zapatista de Liberación Nacional. Enlace zapatista. Recuperado de enlacezapa tista.ezln.org.mx

Suprema Corte de Justicia de la Nación (1939). Calidad de los peones acasillados. En Amparo directo en materia de trabajo 5095/39. México DF, México: SCJN. 